> Une fois n'est pas coutume, la rubrique «Myologie dans le Monde » s'intéresse, non pas à un pays ou à une zone géographique donnée, mais à une population en particulier. Les Tsiganes ou les Roms, appelez-les comme vous voudrez, représentent environ une dizaine de millions d'individus en Europe. Cette population souvent stigmatisée et victime de persécutions, notamment à l'Est de notre Vieux Continent, paie un lourd tribut aux maladies génétiques du fait d'une forte endogamie. Dans ce contexte, les maladies neuromusculaires ne font pas exception. Elles sont fréquentes chez les Roms et souvent associées, pour certaines d'entre elles, à des mutations dites «privées». Depuis une vingtaine d'années, un petit groupe de myologues européens s'intéresse à cette thématique, particulièrement en Bulgarie où, au-delà de la recherche académique, de réelles avancées ont pu être conduites en matière de prise en charge et de prévention. Chemin faisant, il a également été possible de confirmer l'origine géographique de ce peuple nomade et d'imaginer les modalités de ses migrations à travers le monde. <

Les ethnonymes ne manquent pas pour désigner ce groupe d'individus venu, très vraisemblablement, du nord de l'Inde: les Roms, les Tsiganes, les Gitans, les Bohémiens, les Romanichels, les Manouches ou les Gypsies, pour ne reprendre que ceux les plus communément utilisés en Europe. Beaucoup de ces termes restent péjorativement connotés. Leur grande variété illustre d'emblée l'importante diversité de cette population, diversité allant à rebours de nos préjugés en la matière. Même s'ils se revendiquent d'un peuple Rom originel, force est de constater leur grand émiettement non seulement géographique mais également culturel, religieux et linguistique.

Vignette (Photo (c) 79491046 : Erica Guilane-Nachez/Fotolia.com).

\section{Myologie et minorités ethniques}

Tous les chemins mènent aux Roms

J. Andoni Urtizberea ${ }^{1}$, Hanns Lochmuller ${ }^{2}$, Ivailo Tournev ${ }^{3}$

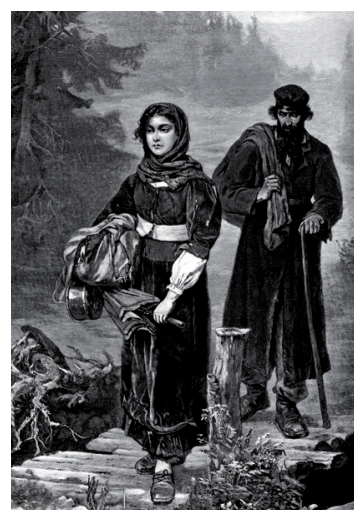

${ }^{1} M D$, Hôpital Marin, Hendaye, France. ${ }^{2} \mathrm{MD}$, PhD, Institute of Genetics, Newcastle, Royaume-Uni. Prof, MD, PhD, DSc,

${ }^{3}$ Department of Neurology, Sofia Medical University, Bulgarie.

\section{Un peu d'histoire}

Il est difficile, faute de documents écrits ou de vestiges archéologiques, de retracer avec précision l'origine et la migration des premiers Roms. Ceux-ci, issus des rangs de groupes nomades du Sind (Pakistan) et du Rajasthan (Inde) auraient quitté le sous-continent indien entre le $v^{e}$ et le $x^{e}$ siècle, possiblement à la demande d'un roi perse. On retrouve ensuite leur trace dans l'empire byzantin aux $x^{e}{ }^{e}$ et $\mathrm{xII}^{\mathrm{e}}$ siècles, notamment dans les Balkans où ils se sont massivement installés avant d'essaimer, pour partie, dans le reste de l'Europe jusqu'au $x v^{e}$ siècle. Trois événements plus récents sont à l'origine de phénomènes migratoires significatifs: l'abolition de l'esclavage des tsiganes en Roumanie à la fin du XIX $x^{e}$ siècle (1868), les conflits en ex-Yougoslavie dans les années 1980, puis les changements liés à l'intégration de nouveaux pays de l'Est au sein de l'Union Européenne, dont la Roumanie et la Bulgarie. L'histoire des origines des Roms reste peuplée de légendes et récits fabuleux comme la migration, jusqu'à présent jamais formellement prouvée, de tsiganes en Espagne par une voie méridionale passant par l'Égypte et l'Afrique du Nord (d'où le terme de « gypsy » faisant clairement référence à l'Égypte, mais terme pouvant aussi être lié à une contrée grecque médiévale du nom de Petite-Égypte où les Roms étaient particulièrement nombreux).

\section{Des groupes culturellement et linguistiquement très disparates}

Fonctionnant au départ un peu comme les jatis indiennes (système de castes), les migrants roms, précisément considérés en Inde comme 


\begin{tabular}{|c|c|c|c|c|c|c|c|}
\hline Disorder & OMIM & Inheritance & $\begin{array}{l}\text { Map } \\
\text { Location }\end{array}$ & Gene & Mutation & Year & $\begin{array}{l}\text { Key } \\
\text { ref. }\end{array}$ \\
\hline Limb girdle muscular dystrophy type $2 \mathrm{C}$ & 253700 & $A R$ & $13 q 12$ & SGCG & C283y & 1996 & [1] \\
\hline Myasthenic syndrome, congenital, type id & 608931 & $A R$ & $17 p 13$ & CHRNE & $1267 \mathrm{delG}$ & 1999 & [2] \\
\hline $\begin{array}{l}\text { Hereditary motor and sensory neuropathy- } \\
\text { Lom }\end{array}$ & 601455 & $A R$ & $8 q 24$ & NDRGl & $\mathrm{R} 148 \mathrm{X}$ & 2000 & [3] \\
\hline $\begin{array}{l}\text { Congenital cataracts facialdysmorphism } \\
\text { neuropathy syndrome }\end{array}$ & 604168 & $A R$ & 18qter & CTDPl & $\begin{array}{l}\text { IVS6 } \\
+389 C>T\end{array}$ & 2003 & [4] \\
\hline GNE myopathy & 603824 & AR & $9 p 13.1$ & GNE & I587T & 2005 & {$[5]$} \\
\hline Charcot-Marie-Tooth disease, type $4 \mathrm{C}$ & 601596 & $A R$ & $5 q 32$ & SH3TC2 & R1109X & 2005 & [6] \\
\hline $\begin{array}{l}\text { Hereditary motor and sensory neuropathy- } \\
\text { Russe }\end{array}$ & 605285 & $A R$ & $10 q 22$ & HKl & $\begin{array}{l}\mathrm{G}>\mathrm{C} \text { in untrans- } \\
\text { lated AltT2 exon }\end{array}$ & 2009 & [7] \\
\hline
\end{tabular}

Tableau I.

hors-castes (soit l'équivalent des intouchables) ont longtemps été associés à leurs métiers. Par la suite, ils ont très souvent adopté les religions, les coutumes, voire les patronymes des contrées où ils se déplaçaient ou dans lesquelles ils s'étaient fixés (comme en Espagne). D'où cette grande diversité que l'on observe y compris au niveau linguistique. Même si les Roms revendiquent, à juste titre, leur attachement à une langue commune, le romani (une langue indo-européenne dérivée du sanskrit), on ne compte pas moins de 100 dialectes roms différents avec de nombreux emprunts dans les langues du MoyenOrient (perse, arménien, turc ou kurde). Selon Jean-Pierre Liégeois, grand spécialiste européen des tsiganes, ceux-ci forment «une mosaïque de groupes diversifiés et segmentés dont aucun ne saurait représenter un autre ».

\section{Des peuples stigmatisés, exclus et persécutés}

Le passé, mais aussi le quotidien actuel, des populations roms en Europe est caractérisé par le rejet et l'exclusion. En Roumanie, l'esclavage a été la règle pendant 600 ans, jusqu'à son abolition en 1868 . Près de 200000 Roms, selon les historiens de la Shoah, ont été victimes de l'Holocauste pendant la deuxième guerre mondiale et ce, dans l'indifférence générale. Quant à l'Union Européenne, elle reste bien impuissante dans ses incitations, pourtant généreusement financées, à mieux intégrer ces populations confrontées à la montée actuelle des populismes et de la xénophobie.

\section{Roms et maladies neuromusculaires}

Dès les années 1990, plusieurs spécialistes européens de pathologie musculaire se sont faits l'écho d'une fréquence élevée de myopathies et de neuropathies héréditaires dans la population rom. À l'époque, le focus était mis sur l'identification des gènes de maladies neuromusculaires en général et de ce point de vue, les communautés très endogames intéressaient fortement les généticiens. Parmi les dystrophies musculaires des ceintures (LGMD) qui firent l'objet de nombreuses études de cartographie, la sarcoglycanopathie de type gamma (LGMD 2C) a été rapportée avec une grande fréquence dans plusieurs familles roms réparties à travers toute l'Europe (Espagne, Portugal, Italie, France, Suisse, Allemagne et Bulgarie). Dans chacune de ces familles, on notait la présence, majoritairement à l'état homozygote, de la mutation C283y du gène SGCG porté par le chromosome 13. Cette mutation a pu être datée et remonterait à l'an mille.

Au même moment, un groupe très actif de chercheurs et de cliniciens bulgares (Kalaydjieva, Tournev, Guergueltcheva) s'intéressaient, entre autres génopathies fréquentes dans la communauté rom, aux neuropathies sensitivo-motrices héréditaires. Suite à un remarquable travail de terrain conduit avec un neurologue anglais de renom, P.K. Thomas, leurs travaux aboutiront à la découverte de deux formes autosomiques récessives de maladies de Charcot-Marie-Tooth (CMT-Lom et CMTRusse, ainsi appelées en référence aux villes de Bulgarie où elles ont été rapportées pour la première fois) et à la description d'un nouveau syndrome associant cataracte, dysmorphie faciale, retard mental et neuropathie (CCFDN) et à l'identification de trois nouveaux gènes. Dans chacune des trois entités, des mutations uniques ont été 
mises en évidence (voir Tableau /), preuve de leur caractère fondateur au sein de cette population si particulière. Au cours de leurs recherches, nos collègues bulgares ont également mis en évidence une mutation propre aux Roms dans la sous-unité epsilon du récepteur à l'acétylcholine. Cette mutation responsable d'un syndrome myasthénique congénital a été identifiée, grâce au concours de l'équipe de l'Institut Friedrich Bauer de Munich (Lochmüller, Abicht, Walter), dans d'autres communautés roms, notamment en ex-Yougoslavie et en Allemagne. Plus récemment, des travaux, en cours de publication, font état d'une fréquence élevée de myopathie GNE en Bulgarie (près de 50 patients, soit plus qu'en France) avec une mutation là aussi unique (I587T). Il en est de même pour un syndrome associant, au sein d'une grande famille rom hongroise, une atteinte de la corne antérieure avec hypoplasie cérébelleuse et hypomyélinisation, le tout en lien avec des anomalies du gène EXOSC8.

Toutes ces découvertes à travers l'Europe ont logiquement conduit à la formation d'un consortium transnational, sous l'égide et avec le soutien logistique de l'European Neuromuscular Center (ENMC). Sous la présidence de Luciano Merlini (Bologne), plusieurs séminaires ont ainsi réuni cliniciens et chercheurs afin d'établir des critères diagnostiques, tant cliniques que génétiques et des recommandations pour la constitution de cohortes de patients, pour la prise en charge et le conseil génétique.

Si l'inventaire des gènes de pathologies neuromusculaires semble actuellement partiellement clos, d'autres gènes ont été récemment identifiés dans d'autres pathologies neurodégénératives. C'est le cas notamment pour certaines formes d'ataxie spinocérébelleuse (mutation du gène ANO10).

\section{La situation en France}

En France, les équipes de Garches (Barois), de Poitiers (Rideau) et de Strasbourg (Warter) ont été particulièrement impliquées au début de la saga de la mutation C283y, en lien avec le laboratoire de génétique de Cochin (Kaplan) et le laboratoire du Fer à Moulin (Fardeau, Tomé). Par l'entremise de Jean-Pierre Liégeois, le sociologue français dirigeant à cette époque le Centre Européen de Recherches Tsiganes à Paris, des contacts ont été établis avec les dirigeants roms français, notamment ceux de la communauté évangélique, pour essayer de sensibiliser leurs co-religionnaires (mais aussi les autres Roms) à un dépistage précoce de cette myopathie et à un conseil génétique approprié.

Outre les cas de LGMD2C, on notera qu'un patient atteint de CCFDN a été diagnostiqué à Marseille, tout comme quelques cas de HSMN-Lom dans une famille ayant migré depuis la Roumanie.

\section{La Bulgarie, un exemple à suivre?}

Même si la population rom y reste encore majoritairement discriminée et marginalisée, des actions de santé publique innovantes et efficaces ont été conduites en Bulgarie où cette communauté représente entre 600 et 800000 personnes, soit $9 \%$ de la population bulgare. À l'initiative des autorités sanitaires et des myologues de Sofia et en accord avec les intéressés, des enquêtes épidémiologiques de grande ampleur

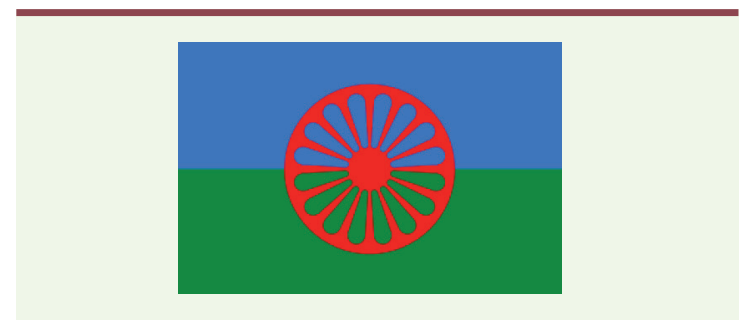

Le drapeau « officiel » de la communauté Rom adopté en 1971.

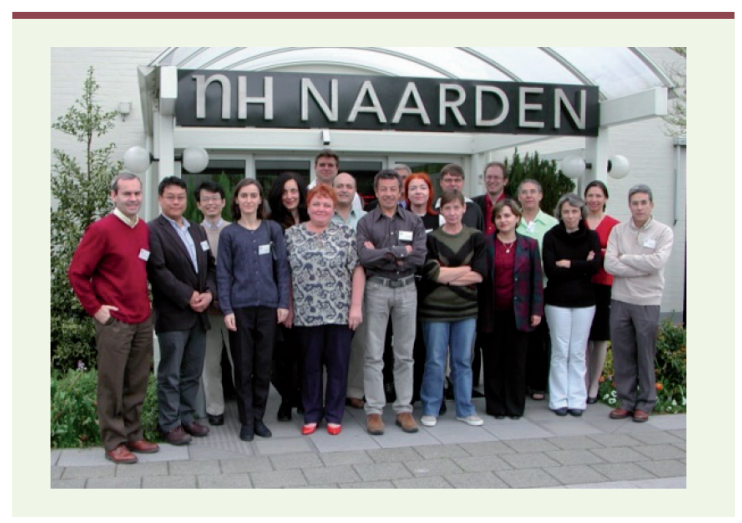

Le Consortium ENMC - Gypsies - Naarden, 2004.

se sont déroulées sur le terrain (technique du «porte à porte », échantillons sanguins) et se sont étalées sur 20 ans. Pas moins de 5500 personnes de la communauté rom ont ainsi pu être génotypées au cours de ces différentes campagnes. Elles ont pu démontrer l'amplitude du phénomène d'endogamie et du risque génétique, particulièrement dans le domaine des maladies neuromusculaires citées plus haut.

En quelques années, 170 médiateurs de santé issus des rangs de la communauté rom ont été formés à travers tout le pays et font désormais le lien avec les structures sanitaires en place, qu'il s'agisse d'actions de prévention ou de conscientisation dans le domaine de la santé. Des études plus ciblées sur les pathologies neuromusculaires citées plus haut ont également permis d'identifier des sous-groupes roms très à risque et dans lesquels la fréquence des hétérozygotes peut atteindre jusqu'à $20 \%$. On notera enfin que près de 75 diagnostics prénataux ont été réalisés pour les seules maladies neuromusculaires (dont l'amyotrophie spinale infantile) dans cette population-cible.

\section{Retour aux racines}

La question de l'origine des roms est toujours restée prégnante au cours de ces travaux de recherche. L'hypothèse, assez simpliste il est vrai, que nous formulions était que de telles mutations pathogènes devraient se 


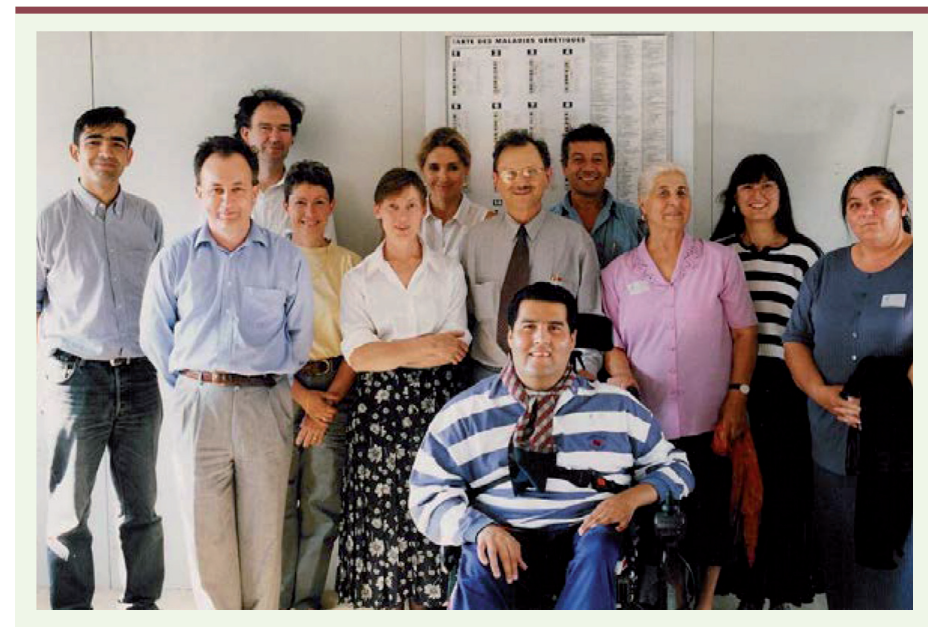

Rocco (atteint de LGMD 2C), sa famille et une partie du Consortium International «Gypsy Genetic Heritage » (où l'on reconnaitra pêle-mêle M. Yanes, M. Jeanpierre, J.-P. Liégeois, D. Récan, L. Kalaydjieva, F. Leturcq, J.-C. Kaplan, L. Merlini, F. Piccolo) (photo prise en 1998).

retrouver chez des patients diagnostiqués et vivant en Inde. Grâce aux contacts établis avec nos homologues de Mumbai (Khadilkar), Delhi (Kalra), Bangalore (Nalini) et Chennai (Vishwanathan), notre quête a été en partie couronnée de succès. À ce jour et sous réserve d'inventaire, un lien formel n'a pu être établi que pour deux pathologies seulement: le syndrome myasthénique congénital avec mutation du gène CHRNE (patients identifiés en Inde du Sud [Vishwanathan]) et plus récemment, la myopathie GNE (mutation fondatrice p.lle587Thr bulgare identifiée dans deux familles originaires du Rajasthan [Khadilkar) portant la même mutation I587T que les patients tsiganes identifiés en Bulgarie et génotypés à l'aide du laboratoire allemand d'Essen [Cirak, Voit]). Pour les autres pathologies (LGMD2C, HSMN-L, HSMN-R ou CCFDN), on peut faire l'hypothèse que l'événement mutationnel fondateur s'est produit soit après la migration des roms hors du sous-continent indien, soit que ces maladies neuromusculaires ne sont pas encore suffisamment reconnues et donc diagnostiquées làbas. Chaque fois que possible, les mutations ont pu être datées à l'aide d'une analyse des haplotypes.

\section{Des défis en grand nombre}

De nombreux défis attendent les Roms tout comme les chercheurs et cliniciens qui se préoccupent de leur sort. Outre l'enjeu sociétal déjà cité et sans doute prioritaire, l'importance de l'endogamie et des maladies génétiques passent par l'éducation, la prévention et la diffusion des connaissances.

D'un point de vue plus académique, plusieurs travaux de recherche seraient à mener. Le travail d'inventaire des maladies neuromusculaires chez les populations roms reste à faire ou à parfaire dans les pays proches de la Bulgarie, particulièrement en Roumanie et en Turquie et au-delà en Iran (terre de passage obligatoire où subsistent quelques groupes roms nomades du nom de Zargars). En Roumanie, les Roms sont

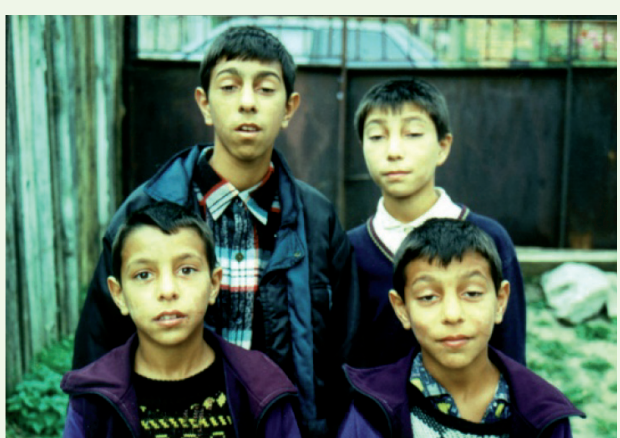

Une fratrie rom de l'Europe de l'Est atteinte d'un syndrome myasthénique congénital de type la.

encore très nombreux (jusqu'à 2 millions). Des premières études avaient été conduites en collaboration avec l'équipe italienne de Bologne (Merlini), mais n'ont pas été aussi abouties et productives qu'en Bulgarie.

On pourra également s'intéresser aux deux extrémités des trajectoires migratoires. L'Inde commence à se doter d'outils diagnostiques performants en matière de génotypage. Il est vraisemblable que d'autres malades indiens seront porteurs des mutations roms déjà décrites. Restera aussi à retracer le parcours en Inde des Roms originels. Ceux-ci ont en Inde plusieurs appellations : Lovaris, Lambadis, Pandjaras. Tous ne sont pas partis vers l'Ouest, loin s'en faut. Ils ont essaimé dans le reste de l'immense territoire indien où ils restent, eux aussi, largement stigmatisés. II nous faudra regarder aussi vers l'Ouest mais au-delà de l'Europe, car un nombre non négligeable de Roms ont émigré vers le Nouveau-Monde (souvent contraints et forcés, il faut le dire).

La question des essais thérapeutiques se posera aussi à un moment ou un autre. Nous pensons particulièrement à la myopathie GNE où le fort contingent bulgare pourrait idéalement avoir accès à la supplémentation en acide sialique retard en phase de test. $\diamond$

Myology and ethnic minorities: all roads lead to the Roma

\section{LIENS D'INTÉRÊT}

Les auteurs déclarent n'avoir aucun lien d'intérêt concernant les données publiées dans cet article.

\section{RÉFÉRENCES}

1. Piccolo F, Jeanpierre M, Leturcq F, et al. A founder mutation in the gammasarcoglycan gene of Gypsies possibly predating their migration out of India. Hum Mol Genet 1996 ; $5: 2019-22$.

2. Abicht A, Stucka R, Karcagi V, et al. A common mutation (epsilon1267delG) in congenital myasthenic patients of Gypsy ethnic origin. Neurology 1999 ; $53: 1564-9$. 


\section{RÉFÉRENCES}

3. Kalaydjieva L, Gresham D, Gooding R, et al. N-myc downstream-regulated gene 1 is mutated in hereditary motor and sensory neuropathy-Lom. Am J Hum Genet $2000 ; 67:$ 47-58.

4. Varon R, Gooding R, Steglich C, et al. Partial deficiency of the C-terminal-domain phosphatase of RNA polymerase II is associated with congenital cataracts facial dysmorphism neuropathy syndrome. Nat Genet $2003 ; 35: 185-9$.

5. Cirak S, Tournev I, Herrmann R, et al. An autosomal recessive distal myopathy with cardiac involvement due to missense mutations in the kinase domain of the UDP-N-acetylglucosamine 2 -epimerase/ $\mathrm{N}$-acetylmannosamine kinase (GNE) gene. Neuropediatrics 2005 ; 36-V3 (Congress abstract).
6. Gooding R, Colomer J, King R, et al. A novel Gypsy founder mutation, pArg1109X in the CMT4C gene, causes variable peripheral neuropathy phenotypes. J Med Genet $2005 ; 42$ : e69.

7. Hantke J, Chandler D, King R, et al. A mutation in an alternative untranslated exon of hexokinase $l$ associated with hereditary motor and sensory neuropathy-Russe (HMSNR). EurJ Hum Genet 2009; 17 : 1606-14.

8. Chamova T, Guergueltcheva V, Gospodinova M, et al. GNE myopathy in Roma patients homozygous for the p.1618T founder mutation. Neuromuscul Disord $2015 ; 25: 713-8$.

TIRÉS À PART

J.A. Urtizberea

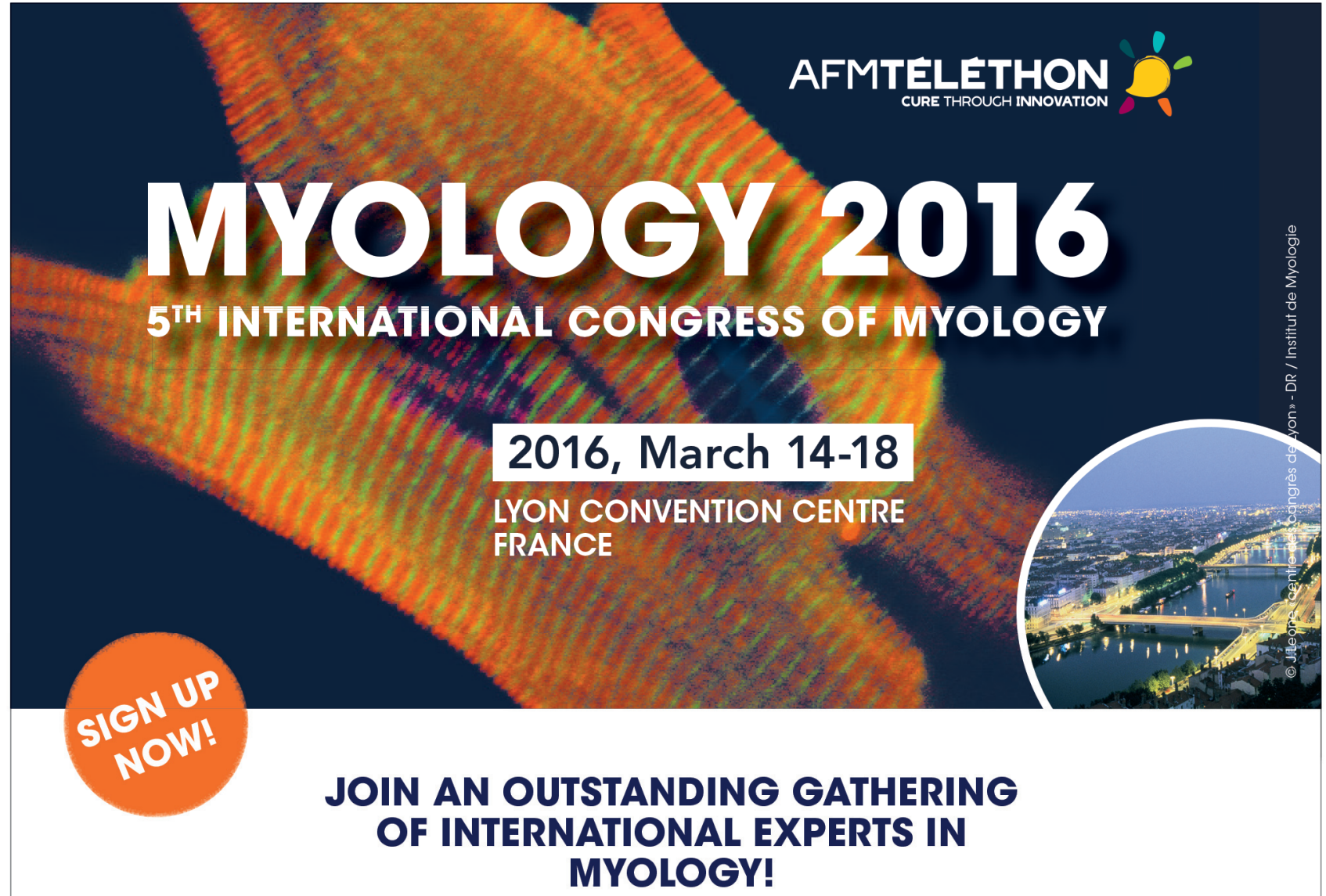

Since the last edition of our congress in 2011, Myology has undoubtedly reached a new milestone: pathophysiological and therapeutic breakthroughs multiply, and clinical trials thrive. The best specialists in this emerging discipline will come from the five continents to present and challenge their latest findings not only in fundamental research but also in clinical science and therapeutics. At Myology 2016, excellence will again be at the rendezvous!

Visit our website

www.myology2016.org

D Registration Early bird fees until January $31^{\text {th }} 2016$ 